\title{
Contribuição de Eletrocardiografia Dinâmica pelo sistema Holter na Avaliação de Pacientes com a Síndrome Congênita do QT Longo
}

\author{
Ivan G. Maia, Marcio L. A. Fagundes, Fernando E. S. Cruz Fo, Rosa Célia P. Barbosa, \\ Paulo A. G. Alves
}

Rio de Janeiro, RJ

\begin{abstract}
Objetivo - Avaliar pelo Holter-24h a dinâmica da repolarização ventricular de pacientes com a sindrome congênita do QT longo.

Métodos - Foram incluidos seis pacientes, sendo os resultados confrontados com os observados em um grupo controle semelhante em número, idade e sexo. Analisaramse nas gravações a morfologia da onda Te sua dinâmica, $o$ intervalo QTc médio para as $24 \mathrm{~h}$, as relações entre $Q T$ e ciclo cardíaco, a variabilidade do QTc das $24 \mathrm{~h}$, bem como a sua dispersão (diferença entre o maior e menor QTc observado). Todas as variáveis foram definidas a partir da medida do intervalo QT obtida no $1^{\circ}$ minuto de cada hora da gravação.
\end{abstract}

Resultados - Alterações morfológicas da onda Testiveram presentes em todos os pacientes, observando-se em cinco deles freqüentes episódios de alternância da onda T, achados ausentes no controle. QTc médio para as $24 \mathrm{~h}$

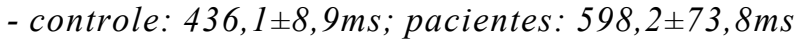
$(p=0,000)$. Correlação linear entre QT e ciclo cardíaco - controle: $r=0,967$; pacientes: $r=0,812(p=0,000)$. Vari-

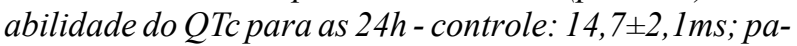
cientes: $36,9 \pm 17,2 \mathrm{~ms}(p=0,01)$. Dispersão do QTc - controle: 53,3 $\pm 8,1 \mathrm{~ms}$; pacientes: $168,3 \pm 70,2 \mathrm{~ms}(p=0,000)$.

Conclusão - Os resultados apresentaram ampla variabilidade dos intervalos QTc nas 24h, menor adaptabilidade do intervalo QT às flutuações dos ciclos cardíacos e significativa dispersão da repolarização ventricular nas 24h, confirmando mais uma vez a existência de importante alteração da vulnerabilidade ventricular nesses pacientes.

Palavras-chave: Holter, síndrome congênita do QT longo, intervalo QT

\section{Contribution of the Dynamic Eletrocardiography by Holter Monitoring in the Evaluation of Congenital Long QT Syndrome Patients}

Purpose - The purpose of this study was to evaluate the value of ambulatory electrocardiogram as a clinical tool to assess ventricular repolarization in patients with the congenital long QT syndrome.

Methods - The study population comprised six patients and their data were compared to a control group of six patients matched in age and gender. The QT interval (ms), corrected by the heart rate, was measured in the first minute of each hour using two monitoring leads, with the mean of six consecutives complexes. The data obtained include the morphologic pattern of T wave, the mean 24-h QTc interval, relation between $Q T$ and cardiac cycle, QTc variability (assessed calculating hourly standard deviation of the interval and then obtaining the global 24-h mean), QTc dispersion (difference between the longest and shortest QTc interval).

Results - In all patients abnormal patterns of T waves were detected with frequent episodes of T wave alternans. Mean 24-h QTc-patients: 598.2 $\pm 73.8 \mathrm{~ms}$; controls: $436.1 \pm 8.9 \mathrm{~ms}(p=0.000)$. Linear correlation and regression between QT and heart rate-patients: $r=0.812$; controls: $r=$ $0.967(p=0.000)$. QTc variability - patients: $36.9 \pm 17.2 \mathrm{~ms}$; controls: $14.7 \pm 2.1 \mathrm{~ms}(p=0.01)$. QTc dispersion - patients: 168.3 $\pm 70.2 \mathrm{~ms}$; controls: $53.3 \pm 8.1 \mathrm{~ms}$ ( $p=0.000)$.

Conclusion - The data showed increased hourly QTC variability, QTc dispersion and worse correlation between QT and heart rate. This data may reflect an abnormally augmented ventricular vulnerability.

Key-words: Holter, congenital long QT syndrome, QT interval

Arq Bras Cardiol, volume $71\left(n^{\circ} 1\right), 49-54,1998$

Hospital Pró-Cardíaco/Pró-Ritmo/Ritmolab/Pró-Criança - Rio de Janeiro Correspondência: Ivan G. Maia - Rua Raul Kennedy 81 - 22631-200 - Rio de Janeiro, RJ

Recebido para publicação em 19/2/98

Aceito em 15/4/98
Contribuições através de ensaios multicêntricos, sofisticação metodológica para o diagnóstico com um auxílio multidisciplinar ${ }^{1-5}$, mudaram profundamente, nestes últimos dez anos, nossos conhecimentos em relação aos mecanismos fisiopatológicos e o perfil clínico envolvidos com a 
síndrome congênita do QT longo (SCQTL). Esses diversos estudos culminaram com o reconhecimento de uma importante base genética para o desenvolvimento da síndrome, identificando-se, atualmente, três genes responsáveis pelas várias formas clínicas de sua apresentação: SQTL1 ligada ao cromossoma 11, SQTL 2, ligada ao cromossoma $7 \mathrm{e}$ SQTL 3 ligada ao cromossoma 3; as duas primeiras comprometendo a dinâmica de específicos canais de potássio e a última a recuperação de canais de sódio ${ }^{6-10}$. Baseados nestas constatações, criou-se a singular possibilidade de serem estabelecidas propostas terapêuticas a partir das relações genotipo/fenotipo ${ }^{11}$.

A eletrocardiografia dinâmica pelo sistema Holter tem se mostrado muito útil na avaliação de vários aspectos clínicos da SCQTL ${ }^{12}$. Os registros contínuos muito contribuíram para a análise da dinâmica da repolarização ventricular, sua morfologia e relações com os ciclos cardíacos, os efeitos da terapêutica, as diferenças nictemerais e influências autonômicas bem como do exercício e estresse. Curiosamente, não encontramos trabalhos em literatura médica de língua portuguesa, avaliando estes diversos aspectos da SCQTL, tendo se tornado o objetivo do presente estudo.

\section{Métodos}

O estudo envolveu seis pacientes, quatro do sexo masculino, idades variando de três a 18 (média 7,3 $\pm 5,9$ ) anos. Segundo Schwatz e col ${ }^{13}$, três pacientes apresentavam pelo menos dois critérios maiores e três outros, um maior e dois menores para o diagnóstico da SQTL (tab. I). Quatro pacientes faziam uso de betabloqueador (propranolol)e um de inibidor da enzima conversora de angiotensina. Por motivos éticos, os registros eletrocardiográficos contínuos foram obtidos na vigência da medicação, invalidando a análise dos dados referentes às variações dos ciclos cardíacos, freqüentemente, alterados nesta população. Em um caso, a terapêutica com betabloqueador foi instituída após a $1^{\mathrm{a}}$ gravação do Holter. Três pacientes apresentavam sequielas neurológicas por parada cardiorrespiratória (PCR) prévia, dois com déficit motor e em uso de cadeira de rodas e o $3^{\circ}$ com déficit mental. Um desses pacientes apresentava aspecto marfanóide, sem diagnóstico definitivo desta condição. Em todos os três pacientes, o diagnóstico da condição foi estabelecido após a PCR. Em um caso, a PCR ocorreu durante procedimento cirúrgico não cardiológico, em outro, dentro de um transporte coletivo e, no último, em via pública. Em todos os pacientes, as gravações de Holter $24 \mathrm{~h}$ foram obtidas após o diagnóstico da SCQTL, estabelecido por cardiopediatras. A identificação dos pacientes encontra-se na tabela I.

Todos os pacientes foram submetidos a pelo menos uma gravação de Holter $24 \mathrm{~h}$, sendo a $1^{\mathrm{a}}$ incluída para o estudo. Utilizaram-se gravadores convencionais e as fitas foram reanalisadas por um único observador em programa desenvolvido pela DMI (Diagnostic Medical Instruments), inserido em um micro-586. As derivações monitoras utilizadas foram a MC1 (semelhante a V1/V2) e MC5 (semelhante a V5).
Alguns registros foram obtidos com três canais de gravação simultâneos, usando-se a derivação aVF modificada para o $3^{\circ}$ canal. Para efeito de análise, foram avaliados os seguintes parâmetros nos registros: morfologia e dinâmica das ondas T, sendo a análise subjetiva e não quantitativa. Além do aspecto morfológico, a análise da dinâmica incluía a constatação ou não da presença de alternância da onda T, classificada como de morfologia ou de polaridade. No $1^{\circ}$ caso, quando ocorriam alterações seqüenciais na voltagem da onda, modificando o seu aspecto, mantendo-se no entanto a mesma polaridade nas derivações analisadas; no $2^{\circ}$, quando as alterações de voltagem eram também acompanhadas de modificações na polaridade da onda em uma seqüência 1:1 ou variável (positividade e negatividade alternantes).

Dinâmica dos intervalos QT e sua variabilidade determinada a cada hora da gravação: os intervalos QT foram avaliados de forma semi-automática e medidos no $1^{\circ}$ minuto de cada hora da gravação. Os resultados foram expressos pela média de cinco ciclos sucessivos, determinados nas duas derivações monitoras (em caso de três derivações simultâneas abandonou-se aVF modificada) e corrigidos pela frequiência cardíaca (média dos cinco ciclos cardíacos imediatamente precedentes aos intervalos QT medidos - QTc - ms), usandose a fórmula proposta por Bazet ${ }^{14}$. Foram construídas curvas de correlação entre as duas variáveis (QT/ciclo cardíaco). A variabilidade do QTc foi determinada, usando-se a média das 24h do desvio padrão desse intervalo, extraída da média de todas as determinações horárias do mesmo e expressa em ms. Avaliaram-se também possíveis diferenças entre os intervalos QTc no período diurno (8-18h) e noturno (24-8h).

\begin{tabular}{|c|c|c|c|}
\hline \multicolumn{4}{|c|}{ Tabela I - Identificação dos pacientes avaliados } \\
\hline Caso & Sexo/idade & Crit.diag. & Medicação \\
\hline 1 & M 3 & 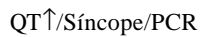 & Propranolol \\
\hline 2 & M 4 & 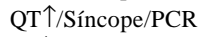 & Propranolol \\
\hline 3 & M 4 & QT $\uparrow / A t l . T / R e p . A n$. & Propranolol \\
\hline 4 & F 18 & $\begin{array}{c}\text { QT } \uparrow \text { Síncope/PCR } \\
\text { (Marfanoide) }\end{array}$ & Inib.ECA \\
\hline 5 & M 11 & 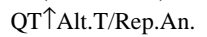 & Nenhuma \\
\hline 6 & F 4 & 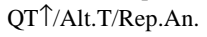 & Propranolol \\
\hline $\begin{array}{l}\text { M- } \\
\text { repol } \\
\text { angic }\end{array}$ & $\begin{array}{l}\text { no; F- fen } \\
\text { lo anormal }\end{array}$ & $\begin{array}{l}\text {; Alt.T- alterações } \\
\text { ECA- inibidor da e }\end{array}$ & $\begin{array}{l}\text { T; Rep.An- } \\
\text { onversora de }\end{array}$ \\
\hline
\end{tabular}

\begin{tabular}{|cccc|}
\hline \multicolumn{4}{|c|}{ Tabela II - Resultados individualizados } \\
\hline Caso & $\begin{array}{c}\text { QTc/24h } \\
(\mathrm{ms})\end{array}$ & $\begin{array}{c}\text { Var.QTc } \\
(\mathrm{ms})\end{array}$ & $\begin{array}{c}\text { Disp.QTc } \\
(\mathrm{ms})\end{array}$ \\
\hline 1 & $682,9 \pm 48,5$ & 48,5 & 200,0 \\
2 & $591,6 \pm 54,8$ & 54,8 & 250,0 \\
3 & $661,6 \pm 59,3$ & 59,3 & 240,0 \\
4 & $497,9 \pm 20,6$ & 20,6 & 90,0 \\
5 & $527,0 \pm 31,8$ & 31,8 & 120,0 \\
6 & $628,3 \pm 27,9$ & 27,9 & 110,0 \\
Média/1DP & $598,2 \pm 73,8$ & $36,9 \pm 17,2$ & $168,3 \pm 70,2$ \\
\hline Var.QTc- variabilidade do intervalo QTc; Disp. QTc- dispersão do inter- \\
valo QTc.
\end{tabular}




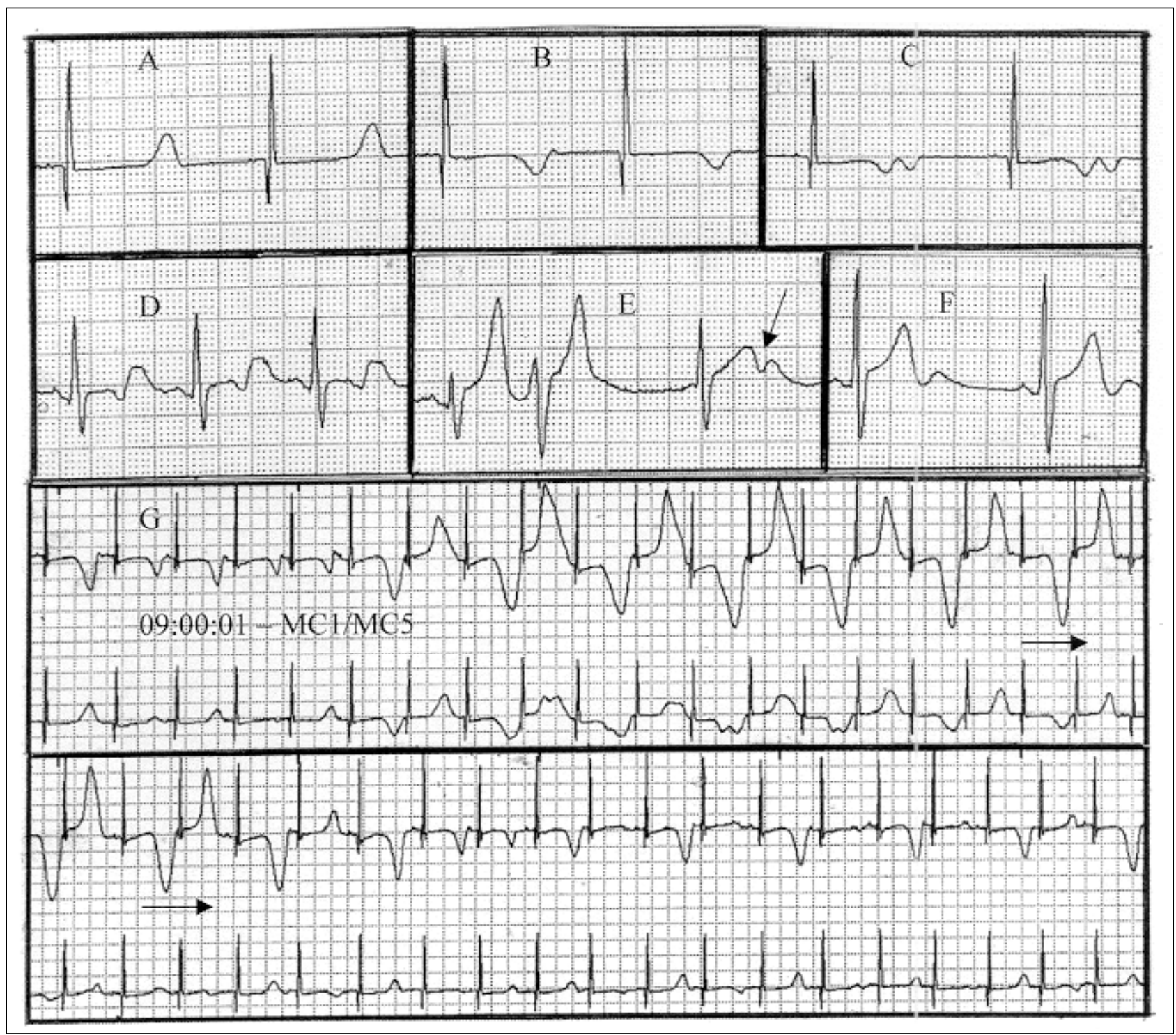

Fig. 1 - Padrões de onda T observados na derivação semelhante a V5 (A-F) e um episódio de alternância da onda T (G - caso 1).

Dispersão do QTc das 24h. O grau de dispersão da repolarização ventricular no período do registro foi determinado através da diferença entre o maior e o menor valor do QTc horário avaliado. Analisou-se também a distribuição dentro do período das $24 \mathrm{~h}$ dos intervalos QTc com maiores graus de dispersão (superiores à média das $24 \mathrm{~h}$ mais um desvio padrão), quantificando-se o grau de dispersão também pelo resultado da subtração com o intervalo mais curto determinado individualmente. Todos os dados foram confrontados com os de um grupo controle semelhante em número, sexo e idade.

Todos os resultados foram expressos pela média e um desvio padrão. Para confronto dos mesmos, utilizou-se um teste de variância com um valor de $\mathrm{p}=0,05$ como significativo. Estabeleceram-se curvas de correlação linear entre os valores dos intervalos QT e seus ciclos cardíacos precedentes.

\section{Resultados}

Morfologia e dinâmica da onda T - Observou-se a existência de um padrão anormal de onda $T$ em todos os pacientes, havendo amplas variações individuais e interindividuais. Os mais característicos foram a presença de entalhes ou uma acentuação do componente terminal da onda, positivo ou negativo (fig. 1). A ocorrência de pausas espontâneas evidenciava ou acentuava esse componente terminal (fig. 1E). Em cinco pacientes foram observados freqüentes episódios de alternância da onda $\mathrm{T}$, sendo mais habitual a do tipo morfológica. A alternância de polaridade foi sempre precedida e sucedida por episódios de alternância morfológica (fig. 1G). Em nenhum dos pacientes do grupo controle ocorreram as diversas alterações descritas.

Dinâmica dos intervalos QTc e sua variabilidade: o intervalo QTc médio para grupo de pacientes e para as $24 \mathrm{~h}$ foi de $598,2 \pm 73,8 \mathrm{~ms}$ com variações de 497,9 a 682,9ms e para o gru- 


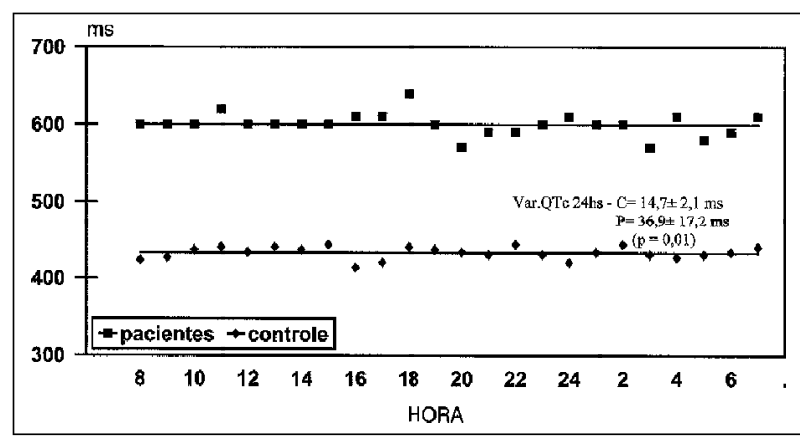

Fig. 2 - Variações horárias dos intervalos QTc sobre a média das 24h nos pacientes e no grupo controle. Var. QTc- variabilidade do intervalo QTc.

po controle de $436,1 \pm 8,9 \mathrm{~ms}$ com variações de $420,0 \mathrm{a} 447,5 \mathrm{~ms}$ $(\mathrm{p}=0,000)$. A figura 2 apresenta as variações horárias dos intervalos QTc em torno da média das $24 \mathrm{~h}$. A curva de correlação linear entre QT horário e ciclos cardíacos mostrou para os pacientes um valor médio de $\mathrm{r}=0,812$ e para o controle um $\mathrm{r}=$ 0,967 ( $\mathrm{p}=0,000$ ) (fig. 3). A variabilidade média dos intervalos QTc dos pacientes encontrava-se de $36,9 \pm 17,2 \mathrm{~ms}$ com variações de 20,6 a 59,3ms. No grupo controle, de 14,7 $\pm 2,1$ com variações de 12,1 a $17,0 \mathrm{~ms}(\mathrm{p}=0,01)$. Não foram observadas nos pacientes diferenças significantes entre a média dos intervalos QTc no período diurno e noturno $(610,0 \pm 80,0 \mathrm{~ms}$ vs $590,0 \pm 71,1 \mathrm{~ms}$ respectivamente, $\mathrm{p}=0,795$ ).

O grau médio de dispersão do QTc para as $24 \mathrm{~h}$ foi para os pacientes de $168,3 \pm 70,2 \mathrm{~ms}$, com variações de 90,0 a $250,0 \mathrm{~ms}$ e no grupo controle de 40 a $60 \mathrm{~ms}$ com uma média de $53,3 \pm 8,1 \mathrm{~ms}(\mathrm{p}=0,000)$. Foram registrados 21 momentos em que a dispersão dos intervalos QTc mostrou-se superior à média individual mais o valor de um desvio padrão, sendo que $14(66,6 \%)$ ocorreram durante o período diurno e sete no período noturno. Na figura 4 mostramos as distribuições horárias desses episódios bem como os seus valores. Esses dados não foram confrontados aos do controle pela sua insignificância nesse grupo (diferenças de 5 a $10 \mathrm{~ms}$ ).

Na tabela II apresentamos os resultados individualizados de todos os parâmetros descritos.

\section{Discussão}

A presença de alterações morfológicas da onda $\mathrm{T}$ tem sido amplamente reconhecida na SCQTL ${ }^{15,16}$, sendo expressão de importantes modificações da repolarização ventricular. Com o Holter, observamos que essas alterações mostraram-se muito dinâmicas e cíclicas nas $24 \mathrm{~h}$ e em um mesmo indivíduo, podendo-se definir como portadores de ondas $\mathrm{T}$ mutantes os pacientes com a SCQTL. Esses achados dificilmente poderiam ser avaliados em registros de curta duração, como os do eletrocardiograma convencional, e certamente encerram aspectos prognósticos importantes.

Recentemente, reconheceu-se que o padrão morfológico da onda $T$ apresenta ligações com marcas genéticas específicas ${ }^{17}$, sendo uma manifestação fenotípica a partir de uma genotípica. Assim, os tipos apresentados na figura $1 \mathrm{~A}, \mathrm{~B} \mathrm{eC}$, em que se observa um início de onda nitidamente

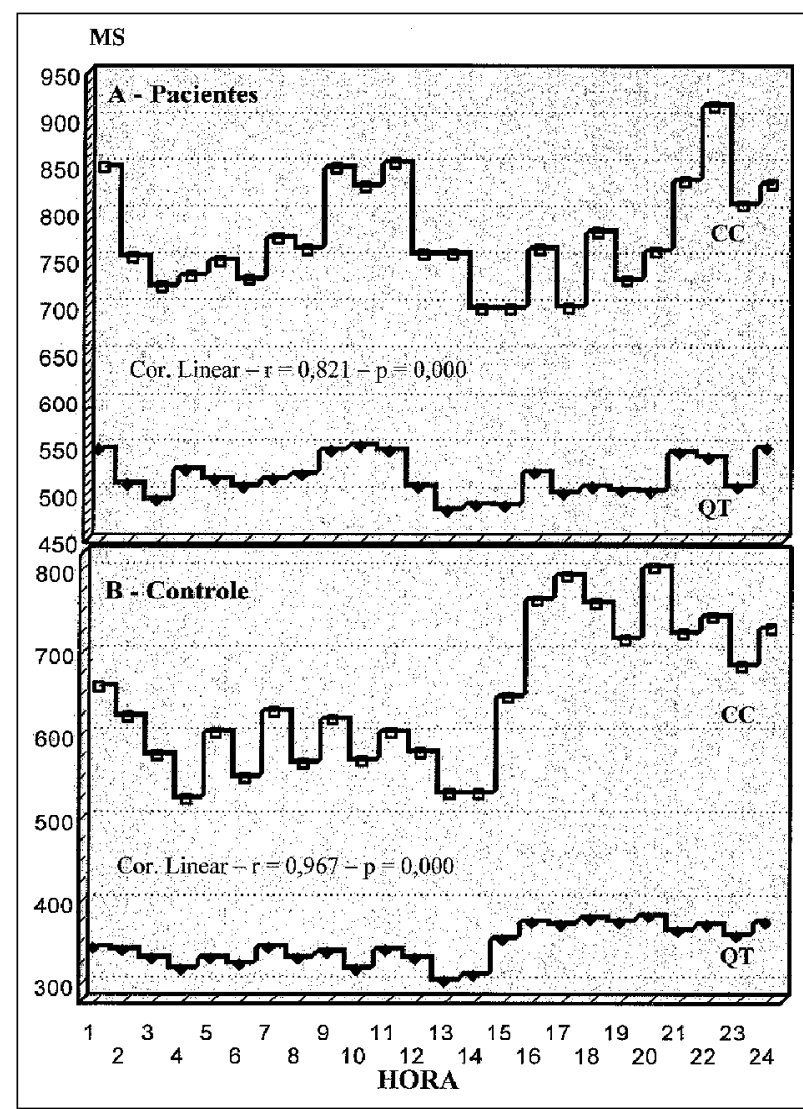

Fig. 3 - Gráfico em degrau mostrando as relações entre ciclo cardíaco e intervalo QT nos pacientes e no grupo controle.

tardio (longo segmento ST) têm sido associados à presença da SCQTL 3, com mutação genética dos canais de sódio. O padrão mostrado na figura $1 \mathrm{EeF}$, com um início de onda bem mais precoce e sua base mais alargada, tem sido mais observado na SCQTL1, ligada à corrente $\mathrm{I}_{\mathrm{KS}}$. Além dessas alterações, aceita-se também, com bases clínicas e experimentais, que a presença de um amplo componente terminal da onda $\mathrm{T}$ (fig. 1E no pós-pausa) seria a expressão eletrocardiográfica de um fenômeno de pós-potencial precoce ${ }^{18}$. Este fenômeno elétrico seria o responsável pela indução de taquicardias polimórficas do tipo torsade de pointes, freqüentemente observadas nessa população específica.

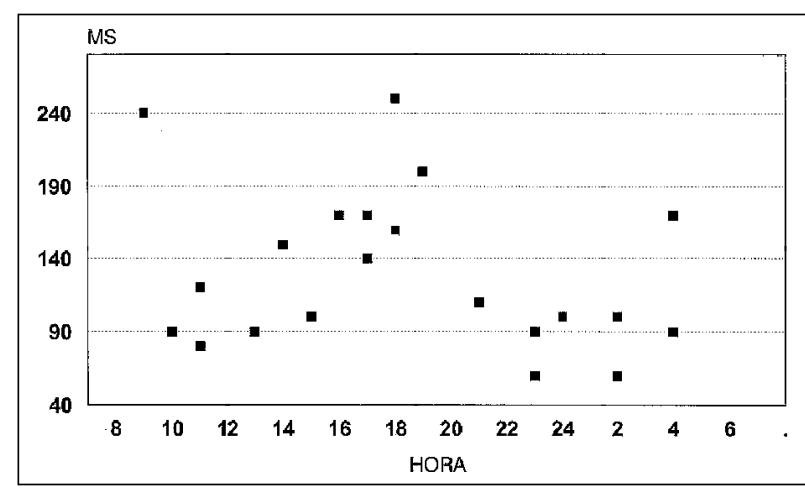

Fig. 4 - Distribuição dentro do período das 24h dos maiores graus individuais de dispersão da repolarização ventricular. Nota-se maior concentração dos episódios entre as $8 \mathrm{e} 22 \mathrm{~h}$. 
Alternância elétrica da onda T poderá ocorrer em níveis imperceptíveis, sendo detectada apenas por técnicas espectrais (micro-alternância) ou se apresentar de forma visível, facilmente observável em um registro eletrocardiográfico (macro-alternância). Sua presença tem importantes implicações prognósticas, sendo um sinal premonitório para o desenvolvimento de arritmias ventriculares graves ou fatais ${ }^{19,20}$. É um achado relativamente freqüente na SCQTL, tendo a mesma conotação clínica ${ }^{21}$. Em nosso material, esteve presente em cinco dos seis pacientes avaliados, manifestandose tanto sob a forma morfológica como de polaridade (fig. 1G). Através do Holter, sua quantificação foi sempre impossível em função do significativo número de vezes em que ocorreue a grande variação na duração dos episódios. Iniciava-se com alguma frequiência associado a um progressivo aumento da frequiência cardíaca, não tendo sido, no entanto, um achado constante. Notamos que as alternâncias de polaridade foram sempre precedidas e sucedidas por um padrão de alternância morfológica. Esses achados sugerem que o processo elétrico gerador do fenômeno, progressivamente se exacerba, até atingir um ápice, retornando posteriormente a sua condição inicial. Distúrbios regionais da repolarização ventricular representariam a base de ligação entre a alternância da onda T e a vulnerabilidade ventricular. Dispersão temporal da repolarização (recuperação não homogênea), associada a um aumento regional da atividade simpática cardíaca, representaria a base para o desenvolvimento da alternância ${ }^{22}$. As alterações de voltagem e de polaridade da onda dependeriam da quantidade de massa miocárdica ventricular dispersa (micro ou macro-alternância), bem como do local de início da recuperação. Quando mais endocárdico produziria ondas T negativas mais epimiocárdico positivas. Na alternância de polaridade, esta relação se apresentaria de forma cíclica 1:1, sendo precedida e sucedida por um progressivo aumento e posterior redução no grau de dispersão. O retardo poderá ser tão significativo a ponto de comprometer a condutividade tissular da despolarização ventricular sucedente, mesmo quando de origem sinusal. A ativação ventricular em momentos de grande dispersão poderia induzir mecanismos reentrantes pelas diferenças regionais da condutividade (condução lenta, bloqueio direcional, anisotropia, etc).

Tem sido demonstrado, através de mapeamento elétrico de superfície, que a região que se projeta sobre a parte frontal esquerda do tórax muito participa na produção da alternância de polaridade ${ }^{23}$.

Os valores do intervalo QT, seu grau de dispersão e variabilidade representam marcadores clínicos importantes para avaliação prognóstica de algumas cardiopatias, incluindo a SCQTL ${ }^{24,25}$. Parece existir uma importante relação entre o grau de dispersão da repolarização ventricular e a morte cardíaca súbita ${ }^{26}$.

Apesar de se ter utilizado técnicas ainda não convencionais, como a determinação da dispersão da repolarização ventricular pelo Holter, compararam-se os resultados avaliados, usando-se as mesmas derivações eletrocardiográficas, porém em momentos distintos (deve-se considerá-la como dispersão horária), e a variabilidade do QTc no início de cada hora (não existe padronização para aquisição desse parâmetro em literatura).Embora existam sugestões de que a determinação dos intervalos QT pelo Holter possam subestimar os resultados ${ }^{27}$, achamos que, apesar dessas limitações, expressaram de forma adequada os propósitos, especialmente, pelas diferenças observadas em relação ao grupo controle. Observamos uma significativa diferença no grau de dispersão da repolarização ventricular e da variabilidade dos intervalos QTc (fig. 2) entre controle e pacientes, como também em relação à adaptabilidade dos intervalos QT aos ciclos cardíacos precedentes (fig. 3). Apenas um paciente (caso 4) mostrou um grau de dispersão inferior a $100 \mathrm{~ms}$, o que certamente expressaria uma profunda alteração nas relações temporais da recuperação ventricular. O mesmo poderia ser dito em relação à variabilidade dos intervalos QTc. Suas amplas flutuações nas $24 \mathrm{~h}$ (associada a um aumento no grau de dispersão) exporia os pacientes a acidentes arrítmicos ventriculares importantes.

Quando distribuímos as maiores dispersões observadas individualmente dentro do período das $24 \mathrm{~h}$, observamos que ocorreram de forma predominante durante a vigília (fig. 4). Acreditamos ser o achado bastante coerente com o padrão de resposta observado na SCQTL. Momentos de estresse, medo, tensão, angústia produzem caracteristicamente, nesta população, importantes modificações na repolarização ventricular ${ }^{28}$, sendo estes acontecimentos teoricamente, mais freqüentes, no período de concentração observado.

O padrão de resposta ou adaptabilidade dos intervalos QT aos ciclos cardíacos representa um importante mecanismo para a estabilidade elétrica miocárdica. Sua redução ou alentecimento poderá comprometer a condutividade tissular. Nossos achados, em relação a essas relações foram semelhantes aos observados por Emori e col $^{29}$ que constataram existir uma modesta correlação entre QT e ciclo cardíaco em portadores da SCQTL. Os mecanismos envolvidos na produção desse fenômeno são desconhecidos, mas certamente, representam mais um fator de aumento da vulnerabilidade miocárdica dos pacientes.

Uma terapêutica adequada, especialmente o uso de betabloqueadores, tende a minimizar as diversas alterações da repolarização, demonstradas e comentadas; inclusive, o grau de dispersão da repolarização ventricular, antes e após o uso desse fármaco, tem sido usado para otimização terapêutica ${ }^{30}$. Na maioria dos nossos pacientes e, apesar do uso desse tipo de droga (dose padronizada de $2 \mathrm{mg} / \mathrm{kg}$ ), mantiveram-se nítidas e muitas vezes proeminentes as modificações cíclicas da repolarização ventricular. Esses achados sugerem, a nosso ver, o uso de doses terapêuticas insuficientes (foi procedido um reajuste na dosagem sem posterior avaliação) ou se tratar de um grupo de pacientes, em sua maioria, não sensíveis aos betabloqueadores em função do tipo genético da doença ${ }^{31}$. Infelizmente não temos condições de avaliar esta última possibilidade.

Em resumo, tentamos demonstrar a importância do Holter na avaliação de pacientes com a SCQTL. Embora usando para quantificação de alguns parâmetros uma metodologia pessoal, pareceu-nos que os resultados foram suficientemente abrangentes para demonstrar a importância do método no estudo desta peculiar e desafiadora síndrome. 


\section{Referências}

1. Schwartz PJ, Periti M, Malliani A - The long QT syndrome. Am Heart J 1975; 89 : 378-90.

2. Schwartz PJ - The idiopathic long QT syndrome-The need for a prospective registry. Eur Heart J 1983; 4: 529-31.

3. Moss AJ, Schwartz PJ, Crampton RS et al - The long QT syndrome: a prospective international study. Circulation 1985; 71: 17-21.

4. Moss AJ, Schwartz PJ, Crampton RS et al - The long QT syndrome: prospective longitudinal study of 328 falilies. Circulation 1991; 84: 1136-44.

5. Roden DM, Lazzara R, Rosen M et al - Multiple mechanisms in the long-QT syndrome. Current knowledge, gaps, and future direction. The SADS Foundation Task Force on LQTS. Circulation 1996; 8: 2012-20.

6. Rosen MR - The long QT syndrome in patients with gene mutations. Circulation 1995; 92: 3373-5.

7. Dumaine R, Wang Q, Keating MT et al - Multiple mechanisms of $\mathrm{Na}^{+}$channellinked long QT syndrome. Circ Res 1996; 78: 916-24.

8. An RH, Bangalore R, Rosero SZ, Kass RS - Lidocaine block of LQT-3 mutan human Na+ channels. Circ Res 1996; 79: 103-8.

9. Priori SG, Napolitano C, Cantu F et al - Differential response to $\mathrm{Na}^{+}$channel blockade, beta-adrenergic stimulation and rapid pacing in a cellular model mimicking the SCN5A and HERG defects present in the long QT syndrome. Circ Res 1996; 78: 1009-15.

10. Mark W, Russel MD, Macdonald Diex II - The molecular genetics of the congenital long QT syndrome. Curr Opin Cardiol 1996; 11: 45-51.

11. Wang DW, Yazawa K, Makita $\mathrm{N}$ et al - Pharmacological targeting of long QT mutant sodium channels. J Clin Invest 1997; 99: 1714-20.

12. Eggeling $\mathrm{T}$, Osterhues $\mathrm{HH}$, Hoeher $\mathrm{M}$ et al - Value of Holter monitoring in patients with the long QT syndrome. Cardiology 1992; 81: 107-14.

13. SchwartzPJ, Moss AJ, Vincent GM, Crampton RS - Diagnostic criteria for the long QR syndrome: an update. Circulation 1993; 88: 782-4.

14. Bazett HC - An analysis of the time relations of electrocardiograms. Heart 1920; 7: 353-70.

15. Garson A Jr, Dick M II, Fournier A et al - The long QT syndrome in children: an international study of 287 patients. Circulation 1993; 87: 1866-72.

16. Lehmann MH, Suzuki F, Fromm BS et al - T wave "humps" as a potential electrocardiographic marker of the long QT syndrome. J Am Coll Cardiol 1994; 24: 746-54.

17. Moss AJ, Zareba W, Benhorin J et al - ECG T wave patterns in genetically distinct forms of the hereditary long QT syndrome. Circulation 1995; 92: 2929-34.
18. Antzelevitch C, Sicouri S - Clinical relevance of cardiac arrhythmias generated by after depolarization: role of $\mathrm{M}$ cells in the generation of $\mathrm{U}$ waves, triggered activity and torsades de pointes. J Am Coll Cardiol 1994; 23: 259-77.

19. Verrier RL, Nearing BD - Electrophysiologic basis for T-wave alternans as an index of vulnerability to ventricular fibrillation. J Cardiovasc Electrophysiol 1994; 5: 445-61.

20. Verrier RL, Zareba W, Nearing BD - T-wave alternans monitoring to assess risk for ventricular tachycardia and fibrillation. In: Moss AJ, Stern S (eds) Noninvasive Electrocardiology. Clinical Aspects of Holter Monitoring. London: Saunders, 1996: 445-64.

21. Malfatto G, Beria G, Sala $S$ et al - Quantitative analysis of $T$ wave abnormalities and their prognostic implications in the idiopathic long QT syndrome. J Am Coll Cardiol 1994; 23: 296-301.

22. Surawicz B, Fisch C - Cardiac alternans: diverse mechanisms and clinical manifestations. J Am Coll Cardiol 1992; 20: 483-99.

23. Shimizu W, Kamakura S, Arakaki Y- T-wave alternans in idiopathic long QT syndrome. Insight from body surface mapping. PACE 1996; 19: 1130-3.

24. Day CP, McComb JM, Campbell RWF - QT dispersion: an indication of arrhythmia risk in patients with long QT intervals. Br Heart J 1990; 63: 342-4.

25. De Ambroggi L, Negroni MS, Monza E et al - Dispersion of ventricular repolarization in the long QT syndrome. Am J Cardiol 1991; 68: 614-20.

26. Zareba W, Moss AJ, le Cessie S - Dispersion of ventricular repolarization and arrhythmic cardiac death in coronary artery disease. Am J Cardiol 1994; 74 550-3.

27. Lubinski A, Lewicka-Nowak E, Kempa M et al - New insight into repolarization abnormalities in patients with congenital long QT syndrome: The increase transmural dispersion of repolarization. PACE 1998; 21(part II): 172-5.

28. Christiansen JL, Guccione P, Garson Jr A - Difference in QT interval measurement on ambulatory ECG compared with standard ECG. PACE 1996; 19: 1296-303.

29. Wellens HJJ, Verneulen A, Durrer D - Ventricular fibrillation ocurring on arousal from sleep by auditory stimuli. Circulation 1972; 46: 661-5.

30. Emori T, Ohe T, Aihara $\mathrm{N}$ et al - Dynamic relationship between the Q-aT interval and heart rate in patients with long QT syndrome during 24-hour Holter ECG. PACE 1995; 18: 1909-18.

31. Priori SG, Napolitano C, Diehl L, Schwartz PJ - Dispersion of the QT interval. A marker of therapeutic efficacy in the idiopathic long QT syndrome. Circulation 1994; 89: 1681-9. 